Supplement of

\title{
Predicting abundance and variability of ice nucleating particles in precipitation at the high-altitude observatory Jungfraujoch
}

\section{Emiliano Stopelli et al.}

Correspondence to: Emiliano Stopelli (emiliano.stopelli@unibas.ch)

The copyright of individual parts of the supplement might differ from the CC-BY 3.0 licence. 


\section{Table of data}

This table presents the most important parameters measured at Jungfraujoch during the 14 sampling campaigns reported in the paper.

$f_{\mathrm{V}}$ represents the fraction of residual water vapour in a precipitating air mass. Season scores correspond to: 1 = winter; 2 = spring; 3 = autumn; 4 = summer. Source region scores correspond to: 1 $=$ north Europe; 2 = mixed; $3=$ south Europe. Values of $N_{>0.5}$ derived from $\mathrm{PM}_{10}$ (as explained in paragraph 2.2) are reported in red.

\begin{tabular}{|c|c|c|c|c|c|c|c|c|c|c|c|c|}
\hline 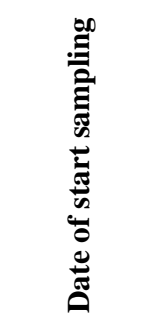 & 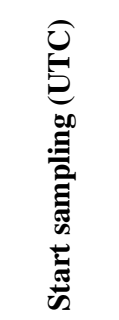 & 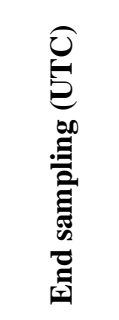 & 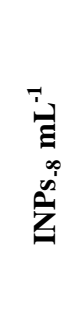 & 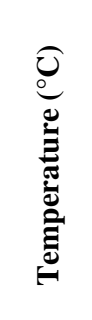 & $\begin{array}{l}3 \\
2 \\
0 \\
0 \\
0 \\
\infty \\
\infty\end{array}$ & $\geq$ & 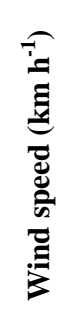 & 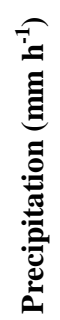 & 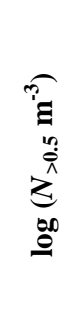 & 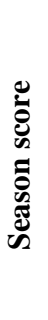 & 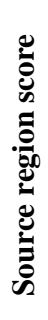 & 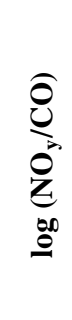 \\
\hline 07.12.2012 & $11.00 \mathrm{~h}$ & $13.15 \mathrm{~h}$ & 0.44 & -15.0 & -32.25 & 0.07 & 32.5 & 2.5 & 4.66 & 1 & 2 & -2.50 \\
\hline 07.12.2012 & $13.20 \mathrm{~h}$ & $15.30 \mathrm{~h}$ & 0.22 & -16.9 & -27.84 & 0.10 & 27.8 & 1.6 & 4.97 & 1 & 2 & -2.34 \\
\hline 07.12.2012 & $15.50 \mathrm{~h}$ & $17.20 \mathrm{~h}$ & 0.22 & -18.9 & -24.48 & 0.13 & 26.2 & 2.2 & 5.17 & 1 & 2 & -2.14 \\
\hline 07.12.2012 & $17.30 \mathrm{~h}$ & 21.00h & 2.11 & -20.5 & -26.90 & 0.11 & 33.9 & 0.4 & 5.22 & 1 & 1 & -2.18 \\
\hline 07.12.2012 & $22.00 \mathrm{~h}$ & $05.30 \mathrm{~h}$ & 0.89 & -20.4 & -31.47 & 0.07 & 37.4 & 1.5 & 4.81 & 1 & 1 & -2.10 \\
\hline 08.12.2012 & $05.30 \mathrm{~h}$ & $09.00 \mathrm{~h}$ & & -20.7 & -30.79 & 0.08 & 32.7 & 3.4 & 5.21 & 1 & 1 & -1.83 \\
\hline 09.12.2012 & $17.15 \mathrm{~h}$ & $19.00 \mathrm{~h}$ & 1.36 & -14.0 & -22.82 & 0.15 & 54.9 & 2.3 & 4.76 & 1 & 2 & -2.59 \\
\hline 09.12.2012 & $19.00 \mathrm{~h}$ & $21.50 \mathrm{~h}$ & 1.61 & -14.5 & -20.96 & 0.18 & 53.6 & 1.1 & 4.93 & 1 & 1 & -2.61 \\
\hline 09.12.2012 & $22.30 \mathrm{~h}$ & 05.00h & 3.49 & -15.9 & -21.29 & 0.17 & 66.2 & 0.4 & 5.05 & 1 & 1 & -2.46 \\
\hline 10.12 .2012 & $05.30 \mathrm{~h}$ & $08.00 \mathrm{~h}$ & 4.09 & -17.8 & -17.00 & 0.25 & 58.3 & 1.8 & 4.82 & 1 & 1 & -2.31 \\
\hline 10.12.2012 & $10.00 \mathrm{~h}$ & $11.00 \mathrm{~h}$ & 2.37 & -19.0 & -18.27 & 0.22 & 50.3 & 4.8 & 4.81 & 1 & 2 & -2.05 \\
\hline 10.12.2012 & $11.15 \mathrm{~h}$ & $13.30 \mathrm{~h}$ & 6.88 & -20.2 & -19.47 & 0.20 & 51.0 & 1.2 & 4.92 & 1 & 2 & -2.13 \\
\hline 10.12.2012 & $14.15 \mathrm{~h}$ & $15.45 \mathrm{~h}$ & 3.78 & -20.7 & -18.44 & 0.22 & 57.6 & 1.8 & 4.90 & 1 & 2 & -2.09 \\
\hline 10.12.2012 & $16.00 \mathrm{~h}$ & $17.30 \mathrm{~h}$ & 4.72 & -20.8 & -19.91 & 0.19 & 57.5 & 3.2 & 6.68 & 1 & 2 & -2.01 \\
\hline 19.01.2013 & $14.00 \mathrm{~h}$ & $16.00 \mathrm{~h}$ & 0.44 & -8.2 & -26.20 & 0.11 & 18.1 & 0.5 & 4.25 & 1 & 3 & -2.45 \\
\hline 19.01.201 & $16.10 \mathrm{~h}$ & $17.30 \mathrm{~h}$ & & -8.7 & -25.07 & 0.12 & 20.9 & 1.1 & 4.45 & 1 & 3 & -2.43 \\
\hline 19.01.2013 & $17.30 \mathrm{~h}$ & $20.00 \mathrm{~h}$ & 1.12 & -8.0 & -22.77 & 0.14 & 31.2 & 0.3 & 4.39 & 1 & 2 & -2.45 \\
\hline 19.01.2013 & $21.00 \mathrm{~h}$ & $05.00 \mathrm{~h}$ & 7.70 & -9.7 & -21.09 & 0.17 & 43.8 & 0.1 & 4.94 & 1 & 3 & -2.31 \\
\hline 20.01.2013 & $05.00 \mathrm{~h}$ & $11.00 \mathrm{~h}$ & 5.34 & -11.0 & -17.35 & 0.23 & 41.6 & 0.1 & 5.69 & 1 & 3 & -2.26 \\
\hline 20.01.2013 & $19.00 \mathrm{~h}$ & $21.00 \mathrm{~h}$ & 2.89 & -13.7 & -17.90 & 0.22 & 45.3 & 0.2 & 4.12 & 1 & 2 & -2.28 \\
\hline 20.01.2013 & $22.00 \mathrm{~h}$ & $04.30 \mathrm{~h}$ & & -13.8 & -19.95 & 0.18 & 38.3 & 0.3 & 3.94 & 1 & 2 & -2.31 \\
\hline 21.01.2013 & $04.30 \mathrm{~h}$ & $08.00 \mathrm{~h}$ & 0.21 & -13.8 & -23.47 & 0.13 & 19.0 & 0.7 & 3.90 & 1 & 2 & -2.36 \\
\hline 09.02.2013 & $02.00 \mathrm{~h}$ & $05.00 \mathrm{~h}$ & 0.43 & -25.8 & -26.06 & 0.12 & 29.4 & 1.4 & 5.00 & 1 & 1 & \\
\hline 09.02 .2013 & $05.00 \mathrm{~h}$ & $07.45 \mathrm{~h}$ & 0.65 & -26.4 & -25.10 & 0.13 & 31.9 & 1.4 & 5.23 & 1 & 1 & -2.05 \\
\hline 09.02.2013 & $07.45 \mathrm{~h}$ & $09.45 \mathrm{~h}$ & 0.21 & -27.0 & -25.82 & 0.12 & 30.7 & 0.9 & 5.23 & 1 & 1 & -2.03 \\
\hline 09.02 .2013 & $09.45 \mathrm{~h}$ & $12.15 \mathrm{~h}$ & 0.21 & -27.1 & -27.50 & 0.11 & 22.6 & 0.7 & 5.44 & 1 & 1 & \\
\hline 09.02.2013 & $14.00 \mathrm{~h}$ & $18.30 \mathrm{~h}$ & & -27.3 & -25.40 & 0.12 & 24.0 & 0.5 & 5.44 & 1 & 1 & -2.00 \\
\hline 10.02 .2013 & $23.00 \mathrm{~h}$ & $05.00 \mathrm{~h}$ & & -14.9 & -30.47 & 0.08 & 12.2 & 0.3 & 4.90 & 1 & 1 & -2.68 \\
\hline
\end{tabular}




\begin{tabular}{|c|c|c|c|c|c|c|c|c|c|c|c|c|}
\hline 11.02 .2013 & $05.00 \mathrm{~h}$ & $09.30 \mathrm{~h}$ & 0.88 & -17.3 & -28.13 & 0.09 & 26.4 & 0.1 & 4.99 & 1 & 2 & -2.38 \\
\hline 11.02 .2013 & $12.00 \mathrm{~h}$ & $14.30 \mathrm{~h}$ & 0.22 & -15.4 & -34.73 & 0.05 & 16.5 & 0.4 & 5.25 & 1 & 2 & -2.37 \\
\hline 11.02 .2013 & $14.30 \mathrm{~h}$ & $17.00 \mathrm{~h}$ & 1.11 & -19.0 & -36.05 & 0.05 & 23.7 & 0.4 & 5.12 & 1 & 2 & -2.37 \\
\hline 11.02 .2013 & $17.00 \mathrm{~h}$ & $21.00 \mathrm{~h}$ & 1.11 & -19.8 & -36.24 & 0.05 & 20.4 & 0.2 & 4.98 & 1 & 2 & -2.32 \\
\hline 11.02 .2013 & $21.30 \mathrm{~h}$ & $04.30 \mathrm{~h}$ & 0.43 & -19.6 & -34.62 & 0.05 & 20.8 & 0.8 & 5.04 & 1 & 2 & -2.27 \\
\hline 12.02 .2013 & $04.30 \mathrm{~h}$ & $07.00 \mathrm{~h}$ & 0.21 & -20.2 & -33.93 & 0.06 & 20.4 & 1.0 & 5.06 & 1 & 2 & -2.16 \\
\hline 10.03.2013 & $23.00 \mathrm{~h}$ & $06.00 \mathrm{~h}$ & 0.21 & -13.6 & -16.38 & 0.27 & 13.0 & 0.1 & 5.49 & 2 & 2 & -2.21 \\
\hline 11.03.2013 & $07.00 \mathrm{~h}$ & $11.00 \mathrm{~h}$ & 0.21 & -12.7 & -17.18 & 0.25 & 10.3 & 0.4 & 4.85 & 2 & 2 & -2.43 \\
\hline 11.03.2013 & $11.00 \mathrm{~h}$ & $15.00 \mathrm{~h}$ & & -12.0 & -17.10 & 0.25 & 1.7 & 0.4 & 4.76 & 2 & 2 & -2.42 \\
\hline 12.03 .2013 & $06.00 \mathrm{~h}$ & $09.30 \mathrm{~h}$ & 0.21 & -13.1 & -22.20 & 0.16 & 6.7 & 0.1 & 4.92 & 2 & 2 & -2.48 \\
\hline 12.03.2013 & $16.00 \mathrm{~h}$ & $18.00 \mathrm{~h}$ & 2.35 & -14.1 & -18.73 & 0.22 & 11.7 & 0.2 & 5.24 & 2 & 2 & -2.17 \\
\hline 13.03.2013 & $21.00 \mathrm{~h}$ & $05.00 \mathrm{~h}$ & 1.35 & -22.4 & -28.01 & 0.10 & 23.8 & 0.2 & 5.18 & 2 & 1 & -1.84 \\
\hline 14.03.2013 & $05.00 \mathrm{~h}$ & $08.00 \mathrm{~h}$ & 2.09 & -24.3 & -29.26 & 0.09 & 38.7 & 0.4 & 5.63 & 2 & 1 & -1.78 \\
\hline 19.04 .2013 & $11.40 \mathrm{~h}$ & $13.40 \mathrm{~h}$ & 1.59 & -6.2 & -9.09 & 0.47 & 19.1 & 2.7 & 6.03 & 2 & 3 & \\
\hline 19.04 .2013 & $13.40 \mathrm{~h}$ & $15.30 \mathrm{~h}$ & 0.21 & -7.1 & -13.48 & 0.35 & 13.7 & 2.4 & 5.26 & 2 & 1 & \\
\hline 19.04 .2013 & $15.30 \mathrm{~h}$ & $18.00 \mathrm{~h}$ & 3.16 & -10.9 & -15.82 & 0.28 & 21.6 & 0.8 & 4.64 & 2 & 1 & \\
\hline 19.04.2013 & $18.00 \mathrm{~h}$ & $20.00 \mathrm{~h}$ & 1.35 & -12.8 & -18.55 & 0.22 & 25.0 & 2.0 & 3.98 & 2 & 1 & \\
\hline 19.04.2013 & $21.30 \mathrm{~h}$ & $04.00 \mathrm{~h}$ & 0.21 & -11.6 & -14.27 & 0.32 & 14.4 & 1.9 & 4.69 & 2 & 2 & \\
\hline 20.04 .2013 & $04.30 \mathrm{~h}$ & $10.30 \mathrm{~h}$ & 8.06 & -10.0 & -12.74 & 0.33 & 56.2 & 0.9 & 4.63 & 2 & 2 & \\
\hline 20.04.2013 & $10.30 \mathrm{~h}$ & $14.30 \mathrm{~h}$ & 0.65 & -8.5 & -14.82 & 0.28 & 48.6 & 0.9 & 4.08 & 2 & 2 & \\
\hline 20.04 .2013 & $14.30 \mathrm{~h}$ & $17.30 \mathrm{~h}$ & 1.35 & -8.1 & -14.95 & 0.27 & 40.7 & 0.9 & 4.03 & 2 & 2 & \\
\hline 20.04 .2013 & $17.30 \mathrm{~h}$ & $19.30 \mathrm{~h}$ & 1.11 & -8.4 & -12.71 & 0.33 & 42.8 & 0.9 & 3.79 & 2 & 2 & \\
\hline 22.05 .2013 & $12.10 \mathrm{~h}$ & $13.45 \mathrm{~h}$ & & -8.4 & -16.55 & 0.26 & 23.8 & 6.3 & 4.30 & 2 & 1 & \\
\hline 22.05 .2013 & $13.45 \mathrm{~h}$ & $15.30 \mathrm{~h}$ & 0.65 & -9.3 & -14.87 & 0.30 & 31.3 & 5.6 & 4.73 & 2 & 1 & \\
\hline 22.05.2013 & $15.30 \mathrm{~h}$ & $17.15 \mathrm{~h}$ & 1.35 & -9.9 & -14.13 & 0.32 & 33.7 & 1.8 & 5.23 & 2 & 1 & \\
\hline 22.05 & $30 \mathrm{~h}$ & 1 & 1 & 0.0 & -14.55 & 0.31 & 33.9 & 2.2 & 4.79 & 2 & 1 & \\
\hline 22.05 .2013 & $21.00 \mathrm{~h}$ & $01.00 \mathrm{~h}$ & 1.11 & -11.3 & -15.27 & 0.29 & 28.0 & 1.4 & 5.05 & 2 & 1 & \\
\hline 23.05.2013 & $30 \mathrm{~h}$ & $15.15 \mathrm{~h}$ & 18.14 & -11.1 & -12.30 & 0.38 & 15.8 & 0.3 & 5.22 & 2 & 1 & \\
\hline 23.05.2013 & $15.15 \mathrm{~h}$ & $16.15 \mathrm{~h}$ & 4.04 & -10.8 & -13.43 & 0.34 & 19.4 & 3.9 & 5.07 & 2 & 1 & \\
\hline 23.05.2013 & $16.15 \mathrm{~h}$ & $17.45 \mathrm{~h}$ & 0.65 & -11.5 & -14.83 & 0.30 & 19.4 & 3.6 & 4.75 & 2 & 1 & \\
\hline 23.05 & $45 \mathrm{~h}$ & $0 \mathrm{~h}$ & 0.43 & -13.2 & -18.14 & 0.23 & 17.1 & 2.1 & 4.90 & 2 & 1 & \\
\hline 23.05 .2013 & $20.45 \mathrm{~h}$ & $04.00 \mathrm{~h}$ & 1.35 & -16.3 & -16.19 & 0.26 & 27.7 & 0.6 & 5.38 & 2 & 1 & \\
\hline 24.05 .2013 & $04.00 \mathrm{~h}$ & 06.00h & 0.88 & -17.2 & -18.76 & 0.21 & 29.5 & 1. & 5.26 & 2 & 1 & \\
\hline 20.06.2013 & $00 \mathrm{~h}$ & $04.00 \mathrm{~h}$ & 113.92 & 0.3 & -4.94 & 0.67 & 66.3 & 0 & 6.37 & 4 & 3 & \\
\hline 20.06.2103 & $04.00 \mathrm{~h}$ & $08.00 \mathrm{~h}$ & 68.09 & -0.1 & -7.14 & 0.55 & 61.9 & 0.7 & 6.27 & 4 & 3 & \\
\hline 20. & $0 \mathrm{~h}$ & 1 & 94.55 & 0.0 & -5.42 & 0.64 & 66.0 & 0 & 6.06 & 4 & 3 & \\
\hline 20.06.2013 & $11.00 \mathrm{~h}$ & $13.30 \mathrm{~h}$ & 182.55 & -0.8 & -9.01 & 0.46 & 75.2 & 0.9 & 6.04 & 4 & 3 & \\
\hline 20.06.2013 & $13.30 \mathrm{~h}$ & $15.30 \mathrm{~h}$ & 159.04 & -1.3 & -8.22 & 0.49 & 89.1 & 0.7 & 5.69 & 4 & 3 & \\
\hline 20.06.2013 & $15.30 \mathrm{~h}$ & $17.30 \mathrm{~h}$ & 72.09 & -1.5 & -8.35 & 0.48 & 74.8 & 1.3 & 5.61 & 4 & 3 & \\
\hline 20.06.2013 & $19.30 \mathrm{~h}$ & $02.00 \mathrm{~h}$ & 16.13 & -1.0 & -7.32 & 0.53 & 40.2 & 1.2 & 5.83 & 4 & 3 & \\
\hline 21.06.2013 & $17.00 \mathrm{~h}$ & $18.30 \mathrm{~h}$ & 56.90 & -0.5 & -5.37 & 0.64 & 15.1 & 0.4 & 5.98 & 4 & 3 & \\
\hline 21.06.2013 & $18.30 \mathrm{~h}$ & $20.00 \mathrm{~h}$ & 10.26 & -0.6 & -6.32 & 0.59 & 11.1 & 1.7 & 5.90 & 4 & 3 & \\
\hline 21.06.2013 & $21.00 \mathrm{~h}$ & $03.30 \mathrm{~h}$ & 0.65 & -2.6 & -12.94 & 0.32 & 12.1 & 2.3 & 5.79 & 4 & 3 & \\
\hline 07.08.2013 & $12.00 \mathrm{~h}$ & $14.00 \mathrm{~h}$ & 122.98 & 0.4 & -3.22 & 0.78 & 59.8 & 0.6 & 6.21 & 4 & 3 & \\
\hline 07.08.2013 & $14.00 \mathrm{~h}$ & $16.10 \mathrm{~h}$ & 68.09 & 0.1 & -5.47 & 0.63 & 65.7 & 1.1 & 5.99 & 4 & 3 & -2.07 \\
\hline 07.08 .2013 & $16.10 \mathrm{~h}$ & $19.20 \mathrm{~h}$ & 434.64 & -0.2 & -5.42 & 0.63 & 76.3 & 0.9 & 6.08 & 4 & 3 & -2.05 \\
\hline
\end{tabular}




\begin{tabular}{|c|c|c|c|c|c|c|c|c|c|c|c|c|}
\hline 07.08.2013 & $20.30 \mathrm{~h}$ & $04.00 \mathrm{~h}$ & 37.44 & 0.2 & -6.99 & 0.54 & 60.0 & 0.7 & 6.25 & 4 & 3 & -2.00 \\
\hline 08.08.2013 & $06.30 \mathrm{~h}$ & $09.30 \mathrm{~h}$ & 7.21 & 0.4 & -8.59 & 0.47 & 30.3 & 0.9 & 6.20 & 4 & 2 & -2.40 \\
\hline 08.08.2013 & $09.30 \mathrm{~h}$ & $11.00 \mathrm{~h}$ & 0.88 & -1.1 & -9.88 & 0.41 & 25.4 & 0.4 & 4.25 & 4 & 2 & -2.23 \\
\hline 08.08.2013 & $11.00 \mathrm{~h}$ & $15.00 \mathrm{~h}$ & 7.21 & -0.4 & -9.29 & 0.43 & 33.6 & 0.9 & 4.62 & 4 & 2 & -2.09 \\
\hline 08.08 .2013 & $15.00 \mathrm{~h}$ & $17.00 \mathrm{~h}$ & 4.04 & 0.2 & -7.50 & 0.52 & 21.0 & 0.3 & 5.40 & 4 & 2 & -2.26 \\
\hline 08.08 .2013 & $20.00 \mathrm{~h}$ & $04.00 \mathrm{~h}$ & 2.61 & -1.3 & -11.89 & 0.34 & 7.7 & 0.2 & 5.15 & 4 & 2 & -2.48 \\
\hline 08.09.2013 & 02.00h & $05.00 \mathrm{~h}$ & 2.35 & -0.8 & -8.78 & 0.47 & 42.4 & 0.9 & 5.56 & 3 & 3 & -2.40 \\
\hline 08.09 .2013 & $12.00 \mathrm{~h}$ & $14.00 \mathrm{~h}$ & 6.39 & -0.1 & -7.78 & 0.52 & 27.6 & 1.4 & 5.30 & 3 & 3 & -2.23 \\
\hline 08.09 .2013 & $14.00 \mathrm{~h}$ & $15.15 \mathrm{~h}$ & 3.86 & -0.4 & -8.33 & 0.49 & 16.0 & 3.0 & 4.96 & 3 & 3 & \\
\hline 08.09 .2013 & $15.15 \mathrm{~h}$ & $16.45 \mathrm{~h}$ & 32.16 & -0.5 & -8.40 & 0.49 & 11.6 & 3.7 & 4.91 & 5 & 3 & -2.30 \\
\hline 08.09.2013 & $17.10 \mathrm{~h}$ & $18.30 \mathrm{~h}$ & 1.11 & -1.0 & -9.00 & 0.46 & 12.6 & 0.7 & 4.94 & 3 & 3 & -2.36 \\
\hline 08.09.2013 & $18.30 \mathrm{~h}$ & $04.00 \mathrm{~h}$ & 3.96 & -3.2 & -14.71 & 0.26 & 16.0 & 1.3 & 4.61 & 3 & 2 & -2.37 \\
\hline 12.09 .2013 & $10.50 \mathrm{~h}$ & $13.50 \mathrm{~h}$ & 1.84 & -7.7 & -9.00 & 0.48 & 17.1 & 0.3 & 4.83 & 3 & 1 & -2.17 \\
\hline 12.09 .2013 & $13.50 \mathrm{~h}$ & $15.40 \mathrm{~h}$ & 2.09 & -7.5 & -11.75 & 0.39 & 14.0 & 0.7 & 4.34 & 3 & 1 & -2.16 \\
\hline 12.09 .2013 & $15.40 \mathrm{~h}$ & $17.00 \mathrm{~h}$ & 1.59 & -7.6 & -13.73 & 0.33 & 17.1 & 1.4 & 4.50 & 3 & 1 & -2.14 \\
\hline 12.09 .2013 & $17.00 \mathrm{~h}$ & $18.30 \mathrm{~h}$ & 3.16 & -8.0 & -13.67 & 0.33 & 18.8 & 0.7 & 4.67 & 3 & 1 & -2.15 \\
\hline 12.0 & $\mathrm{~h}$ & 0 & .89 & -9.2 & -13.33 & 0.34 & 19.8 & 0.2 & 5.10 & 3 & 1 & -2.13 \\
\hline 22.05 .2014 & 04.00h & $12.00 \mathrm{~h}$ & 44.48 & -3.2 & -4.47 & 0.72 & 65.83 & 2.00 & 7.15 & 2 & 3 & -2.13 \\
\hline 22.0 & $12.00 \mathrm{~h}$ & $20.00 \mathrm{~h}$ & 12.01 & -2.8 & -8.81 & 0.49 & 55.30 & 1.95 & 5.74 & 2 & 3 & -2.17 \\
\hline 22.05 .2014 & $20.00 \mathrm{~h}$ & $04.00 \mathrm{~h}$ & 31.51 & -5.1 & -8.01 & 0.52 & 58.68 & 2.32 & 5.48 & 2 & 3 & -2.37 \\
\hline 28.06.2014 & $20.00 \mathrm{~h}$ & $04.00 \mathrm{~h}$ & 5.00 & -1.1 & -9.96 & 0.47 & 26.21 & 0.90 & 5.45 & 4 & 2 & -2.05 \\
\hline 29.06 .2014 & $04.00 \mathrm{~h}$ & $.00 \mathrm{~h}$ & 21 & -1.8 & -14.08 & 0.32 & 9.64 & 1.93 & 4.90 & 4 & 2 & -2.27 \\
\hline 29.06 .2014 & $10.00 \mathrm{~h}$ & $16.00 \mathrm{~h}$ & 6.29 & -2.8 & -12.69 & 0.36 & 14.56 & 1.18 & 4.94 & 4 & 2 & -2.24 \\
\hline 29.06.2014 & $18.00 \mathrm{~h}$ & 04.00h & 2.35 & -7.4 & -14.9 & 0.29 & 34.34 & 0.98 & 5.04 & 4 & 1 & -2.31 \\
\hline 29.07.2014 & 04.00h & 10.00 & 3 & -2.0 & -17.6 & 0.23 & 26.21 & 2.00 & 4.13 & 4 & 2 & -2.24 \\
\hline 29.07 .2014 & $11.00 \mathrm{~h}$ & $15.30 \mathrm{~h}$ & 1.59 & -1.6 & -14.47 & 0.31 & 28.61 & 0.85 & 4.97 & 4 & 2 & \\
\hline 014 & $.00 \mathrm{~h}$ & h & 0.88 & -0.4 & -13.64 & 0.34 & 33.04 & 2.25 & 4.90 & 4 & 1 & -2.22 \\
\hline 30.07 .2014 & $17.00 \mathrm{~h}$ & $01.00 \mathrm{~h}$ & 0.88 & 3.2 & -14.99 & 0.30 & 5.37 & 5.47 & 4.51 & 4 & 1 & -2.22 \\
\hline 30.09 .2014 & $13.00 \mathrm{~h}$ & $13.45 \mathrm{~h}$ & 0.21 & -1.2 & -10.34 & 0.41 & 7.64 & 0.59 & 5.07 & 3 & 3 & -2.22 \\
\hline 01.10 .2014 & 05.00h & 06.30h & 1.59 & -2.5 & -11.17 & 0.38 & 16.99 & 0.73 & 4.70 & 3 & 3 & -2.36 \\
\hline 21.10 .2014 & $20.00 \mathrm{~h}$ & $04.00 \mathrm{~h}$ & 60.51 & -12.8 & -11.46 & 0.39 & 67.95 & 2.00 & 5.35 & 3 & 1 & \\
\hline 22.10 .2014 & 06.00h & 13.00h & 29.02 & -17.0 & -14.72 & 0.29 & 60.40 & 2.00 & 5.23 & 3 & 1 & \\
\hline
\end{tabular}

